\title{
Development of liquid cells for high resolution imaging and chemical analysis in situ with Transmission Electron Microscopy
}

Junyi Shangguang ${ }^{1}$, Xinxing Peng ${ }^{2}$, Sophia Betzler ${ }^{3}$ and Haimei Zheng ${ }^{3}$

${ }^{1}$ University of California, Berkeley, Berkeley, California, United States, ${ }^{2}$ Lawrence Berkeley National

Lab, Berkeley, California, United States, ${ }^{3}$ Lawrence Berkeley National Laboratory, United States

The development of liquid cells for transmission electron microscopy (TEM) have enabled breakthroughs in the study of nanoscale structural, morphological, or chemical changes during materials growth and applications [1]. The challenges of achieving high resolution imaging and chemical analysis through liquids often arise from the liquid cells being too thick. Making ultra-thin freestanding membranes and controlling the liquid thickness are some of the key attributes to the recent liquid cell developments. For example, these were achieved by using low-stress silicon nitride, $\operatorname{SiN}_{x}(x<4 / 3), 15-25 \mathrm{~nm}$ in thickness on a thinner silicon wafer (100 $\mu \mathrm{m}$ thick as compared to the $500 \mu \mathrm{m}$ thick standard wafer) [2-4]. To control the liquid thickness inside the cell, a thin thermally evaporated Sn spacer (70-100 $\mathrm{nm}$ ) was deposited by on the bottom chip of the cell and bottom chip can be bonded with the top chip. The thin wafer design minimizes the attenuation of X-ray signals in energy dispersive X-ray spectroscopy (EDS). Electrochemical liquid cells may be fabricated by incorporating patterned electrodes of various metals (e.g., Au, Ti, and Pt) on the bottom chip of the cell [1]. Superior high-resolution imaging has been accomplished with a range of newer membranes and 2D materials ranging from carbon films [5] to $\mathrm{MoS}_{2}$ combined with graphene [6] (Figure 1a, b).

Recently, we have developed a new type of liquid cells allowing for the studies of chemical reactions in situ and at low temperatures (Figure 1c, d) [7]. It can also accommodate various electron microscopy techniques, such as high resolution (S)TEM imaging, EDS, electron energy loss spectroscopy (EELS) and 4D-STEM at room temperature and from zero to cryo-genic temperatures. Polyimide was selected as the support film on consideration of its high chemical resistance, moderate sensitivity to electron beam and high mechanical stability. Using this type of liquid cells, we have studied the growth of indium chloride $\left(\mathrm{InCl}_{3}\right)$. The nucleation and growth of two-dimensional (2D) nanosheets in solution via both classical and non-classical pathways were observed with atomic resolution in real time at room temperature as well as low temperature (e.g. $1-2{ }^{\circ} \mathrm{C}$ ). We found that the 2D nanosheet undergoes atom-by-atom addition in a layer-by-layer mode, accompanied with in-plane growth and surface atom diffusion. Observations also revealed that the fast growth induced the high density of crystal defects (e.g., nanotwins), which were not obvious in low temperature growth. The atomic structure at the cryogenic temperature provides valuable reference points for this study (Figure 2). The success in the design and fabrication of this new type of liquid cells allowing various low temperatures open the opportunity for investigation of the fine structure of air sensitive functional materials in situ.

Acknowledgements: This work was supported by the U.S. Department of Energy, Office of Science, Offi ce of Basic Energy Sciences, Materials Sciences and Engineering Division, under Contract No. DEAC0205CH11231 within the insitu TEM program $(\mathrm{KC} 22 \mathrm{ZH})$. Work at the Molecular Foundry was supported by the Office of Science, Office of Basic Energy Sciences, of the U.S. Department of Energy under Contract No. DE-AC02-05CH11231. 

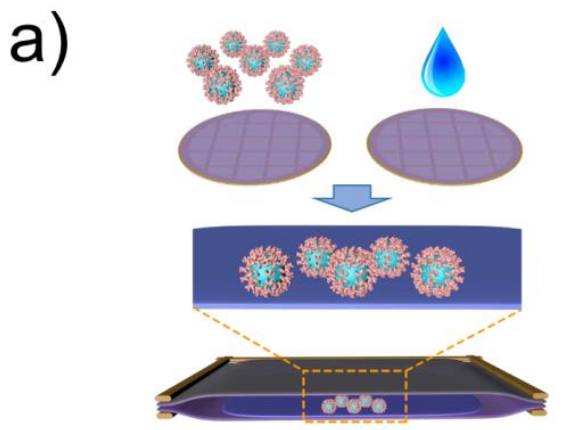

b)

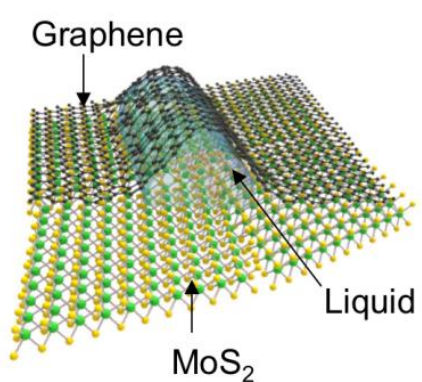

C)
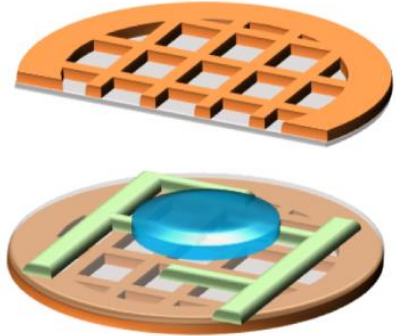

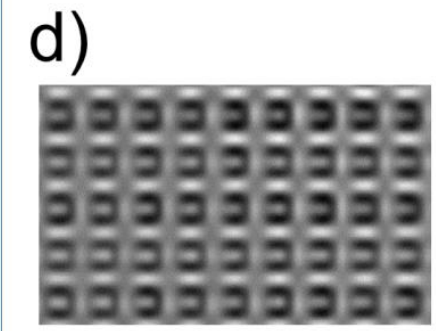

HRTEM

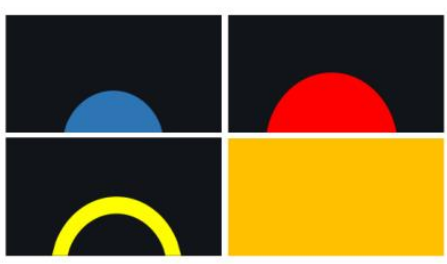

EDS

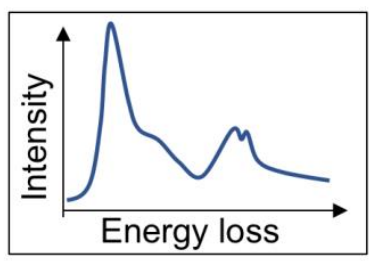

EELS

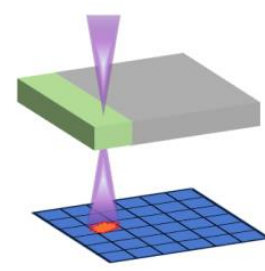

4D-STEM

Figure 1. Figure 1. The development of liquid cells with $2 \mathrm{D}$ membranes allowing high resolution and chemical analysis capabilities. (a) A thin carbon film liquid cells enabling in situ study of nanoparticle self-assembly and superlattice transformations. (b) MoS2 liquid cells. (c) A new electrochemical liquid cell with sandwiched structure (polymer membrane-patterned electrode-polymer membrane). (d) Capability of liquid cells, such as high-resolution TEM imaging, EDS and EELS and 4D-STEM at various temperatures.
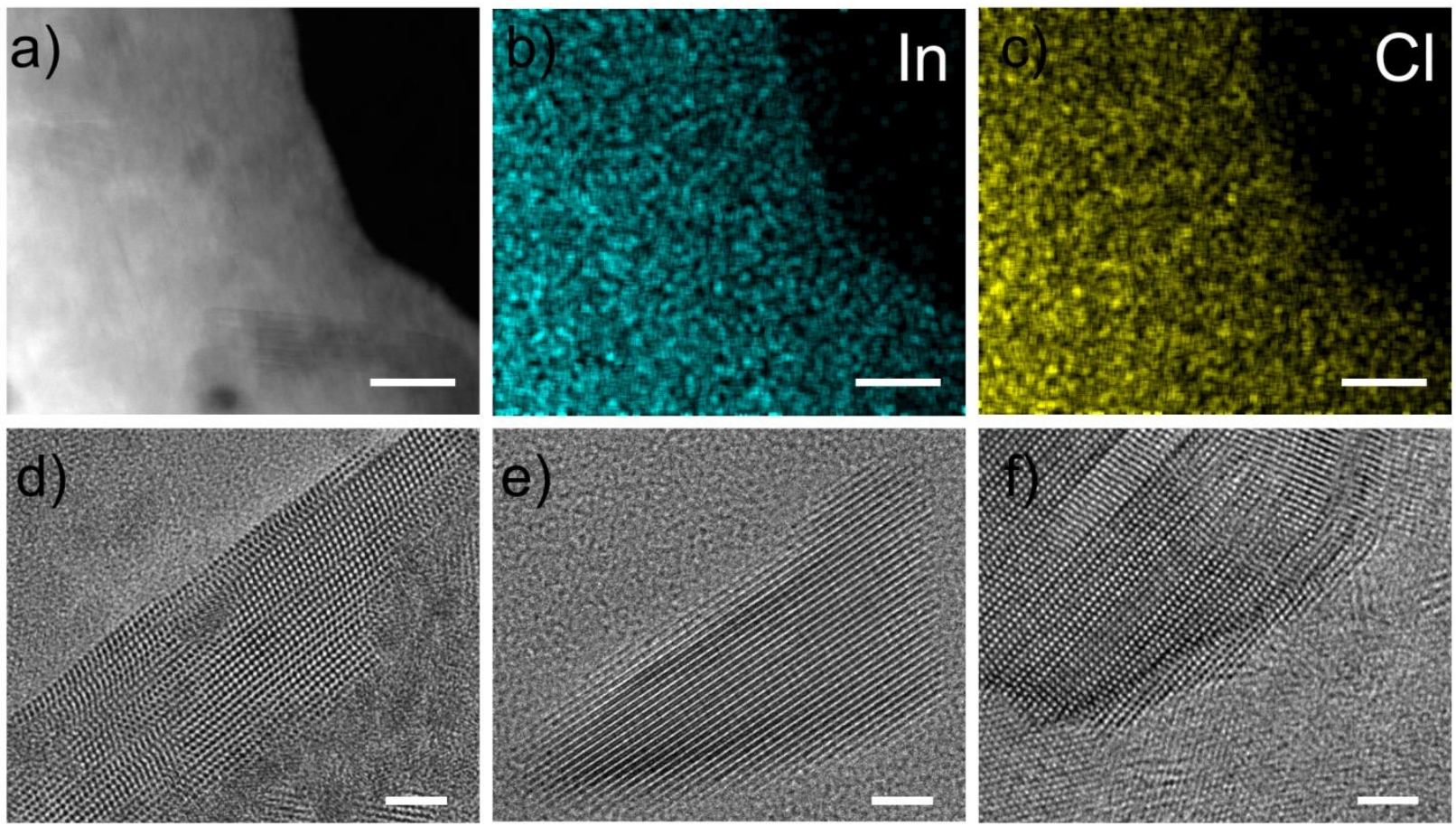

Figure 2. Figure 2. Formation of $\mathrm{InCl} 3$ nanosheets in an aqueous solution at various temperatures. (a) HAADF-STEM image of a representative $\mathrm{InCl} 3$ thin film at cryogenic temperature, (b-c) EDS elemental 
mapping corresponding to (a), scale bars in (a-c) are 20nm. (d) High-resolution TEM images of $\mathrm{InCl} 3$ nanosheets formed at room temperature, (e) $\mathrm{InCl} 3$ nanosheets at $2{ }^{\circ} \mathrm{C}$, (f) $\mathrm{InCl} 3$ at cryogenic temperature, $-180^{\circ} \mathrm{C}$, scale bars in (d-f) are $5 \mathrm{~nm}$.

\section{References}

[1] H. Zheng, MRS Bulletin in press (2021).

[2] H. Zheng et al., Science 324 (2009) p.1309.

[3] H. G. Liao et al., Science 345 (2014) p.916.

[4] H. G. Liao, L. Cui, S. Whitelam and H. Zheng, Science 336 (2012) p.1011.

[5] Y. Wang et al., Science Advances 5 (2019) eaaw5623.

[6] J. Yang et al., Nano Letters 19 (2019) p.1788.

[7] J. Shangguan, X. Peng, S. Betzler, H. Zheng, to be submitted. 